\title{
Bordetella Pertussis Infection in Hospitalized Infants with Acute Bronchiolitis
}

\author{
Şule Gökçe ${ }^{1}$ - Zafer Kurugöl ${ }^{2}$ - S. Şöhret Aydemir ${ }^{3}$ - Candan Çiçek ${ }^{3}$ • Aslı Aslan ${ }^{1}$ • \\ Güldane Koturoğlu ${ }^{1}$
}

Received: 27 February 2017 / Accepted: 5 September 2017 / Published online: 27 October 2017

(C) Dr. K C Chaudhuri Foundation 2017

\begin{abstract}
Objective To assess the frequency of $B$. pertussis infection among young infants hospitalized with acute bronchiolitis and to determine whether $B$. pertussis infection affects the clinical course of acute bronchiolitis.

Methods A total of 172 infants $<6$ months of age hospitalized with acute bronchiolitis were tested for $B$. pertussis and respiratory viruses with real-time PCR. Cases were divided into 2 groups according to $B$. pertussis positive or negative. Clinical parameters, clinical severity scores and laboratory characteristics of the pertussis-positive and pertussis-negative cases were compared.

Results Bordetella pertussis infection was detected in 44 (25.6\%) of the 172 infants hospitalized for acute bronchiolitis, and as co-infection with respiratory viral agents in $27(61.4 \%)$ infants. Of the 44 pertussis-positive infants, only 17 (38.6\%) experienced a paroxysmal cough, $13(29.5 \%)$ had whooping and $15(34.1 \%)$ had post-tussive vomiting. There was no significant difference between pertussis-positive and pertussisnegative infants according to Wang clinical score at admission $(4.9 \pm 1.5$ vs. $5.2 \pm 2.5 ; p=0.689)$. The overall disease severity score was also similar between the two groups $(6.5 \pm 1.4 \mathrm{vs}$. $6.9 \pm 1.6 ; p=0.095)$.
\end{abstract}

Zafer Kurugöl

zafer.kurugol@ege.edu.tr

1 Department of Pediatrics, General Pediatrics Unit, Ege University, Izmir, Bornova, Turkey

2 Division of Pediatric Infection, Department of Pediatrics, Ege University, 35700 Izmir, Bornova, Turkey

3 Department of Microbiology, Ege University, Izmir, Bornova, Turkey
Conclusions Bordetella pertussis infection is common in young infants hospitalized for acute bronchiolitis, mostly as co-infection with respiratory viruses. The clinical features of pertussis in the infants are not characteristic. Viral bronchiolitis and pertussis cases could not be differentiated by clinical findings. Co-infection with pertussis did not affect the clinical outcome in infants hospitalized with acute bronchiolitis.

Keywords Bordetellapertussis · Infant · Acute bronchiolitis · Respiratory viruses $\cdot$ Disease severity score

\section{Introduction}

Acute bronchiolitis is the most common lower respiratory tract infection in infants and young children, and one of the most common causes of hospital admission during infancy. The majority of children hospitalized for bronchiolitis are under the age of 6 mo [1]. It is known that acute bronchiolitis is predominantly a viral disease [2]. Almost $90 \%$ of cases are related to viruses such as respiratory syncytial virus (RSV), adenovirus, coronavirus, parainfluenza, influenza and rhinovirus. In recent years new human respiratory viruses like human metapneumovirus, bocavirus and new human corona viruses have been reported, as possible pathogens causing acute bronchiolitis [3]. Bordetella pertussis has also been identified in infants hospitalized with acute bronchiolitis, as a single or mainly co-pathogen with respiratory viruses [4-10].

In recent years, several studies have been published to assess Bordetella pertussis infection in infants hospitalized for various lower respiratory tract infections including acute bronchiolitis $[4,5,8,10]$. In these studies, some impacts on the disease manifestation were observed in these infants admitted to pediatric wards or the pediatric intensive care unit. However, the clinical significance of Bordetella pertussis 
infection in infants with acute bronchiolitis has not been clearly defined. Therefore, the present study was planned to assess the frequency of Bordetella pertussis infection among infants aged $<6$ mo hospitalized for acute bronchiolitis and to determine whether Bordetella pertussis infection affects the clinical course of acute bronchiolitis.

\section{Material and Methods}

This prospective study was conducted in the Department of Pediatrics, Ege University Hospital (Turkey) between October 2013 and April 2016. Infants younger than 6 mo of age, who were hospitalized for acute bronchiolitis in the General Pediatrics ward of the hospital, were enrolled in the study. The Ethics Committee of the Ege University Hospital approved the study. A written informed consent was obtained from parents before enrolling infants. Acute bronchiolitis was defined as acute lower respiratory illness characterized by rhinorrhea, cough, and diffuse wheezes or crackles [11]. Previously healthy infants $<6$ mo of age, who presented with $\leq 4 \mathrm{~d}$ duration of acute bronchiolitis were included in the study. Infants who were hospitalized within $2 \mathrm{wk}$ before the current admission, those who developed nosocomial acute bronchiolitis, or those who had a known history of bronchopulmonary dysplasia or chronic lung disease or congenital heart disease or any immunodeficiency were excluded.

Detailed demographic, epidemiological and clinical data were recorded on admission and clinical and laboratory data were recorded from each child during the hospitalization. Epidemiological data included age, gender, number of siblings and smoke exposure. Pertussis vaccination status was obtained from the vaccination cards. Presence of characteristic clinical findings of pertussis (paroxysmal cough, whooping, post-tussive vomiting, apne) were recorded on a standard case report form for all patients. On admission, Wang clinical score (composite clinical score with respiratory rate, retraction, wheezing and general condition) was used for the assessment of the severity of acute bronchiolitis [12]. Each clinical sign was scored from zero to 3 except for the general condition, which was scored zero for normal and 3 for irritability or lethargy. The possible total score ranged from 0 to 12 .

An overall disease severity score was also ascertained based on the following parameters: duration of hospital stay, duration of supplemental oxygen therapy, duration of intensive care unit and clinical severity score on admission as previously described by Bamberger et al. [13]. A nasopharyngeal aspirate was obtained from each infant within $24 \mathrm{~h}$ of admission and tested for the presence of respiratory syncytial virus (RSV), influenza virus type $\mathrm{A}$ and $\mathrm{B}$, adenovirus, parainfluenza viruses (types 1-4), human rhinoviruses, human coronaviruses, human metapneumovirus and human bocavirus with real-time multiplex polymerase chain reaction
(PCR) methods (RealAccurate ${ }^{\circ}$, Respiratory RT PCR, PathoFinder, Netherlands and Seeplex ${ }^{\circledR}$ RV15 ACEDetection, Seegene, South Korea).

A second nasopharyngeal sampling was taken easily from each patient by entering through nostrils and rotating the swabs that have flexible, twisted wire shafts with Dacron bud (Transwab, England). The samples were stored at $-80^{\circ} \mathrm{C}$ and were later analyzed at the bacteriology laboratory in the Department of Clinical Microbiology. Pertussis was diagnosed with quantitative multiplex real-time PCR technique which was used on nasopharyngeal samples to amplify targets IS481. Commercial kit (LightMix kits, TIB Molbiol, $\mathrm{GmbH}$, Germany) was used and all the analyses were done by LightCycler 1.5 (Roche Diagnostics, USA).

Statistical analysis was performed using SPSS version 21.0 for personal computers (Chicago, IL, USA). The infants were divided into two groups according to Bordetella pertussis (B. Pertussis) positive or B. pertussis-negative. The two groups were compared in terms of demographic, clinical and laboratory characteristics. Group differences in categorical data were analyzed using the Chi-square test. In cases of nonnormally distributed data, the Mann-Whitney U test was used to determine whether the difference between the two groups was statistically significant. A value of $p<0.05$ was considered as statistically significant.

\section{Results}

A total of 172 previously healthy infants $<6$ mo of age, who were hospitalized for acute bronchiolitis, were enrolled in the study. The mean age of the infants was $11.9 \pm 6.5 \mathrm{wk}$ (range 0-25 wk), and 103 (59.9\%) were male. Fifty-two (30.2\%) infants had received one dose of acellular pertussis vaccine, and $46(26.7 \%)$ had received two doses. Seventy-four (43.1\%) infants aged $<2$ mo were non-vaccinated. Bordetella pertussis was identified in 44 (25.6\%) of the 172 infants hospitalized for acute bronchiolitis (Table 1). Of the 44 pertussis-positive infants, 27 (61.4\%) co-infected with viral respiratory agents, 17 (38.6\%) had only B. pertussis, as a sole pathogen. The most prevalent viral agent was RSV, detected in $88(51.1 \%)$ infants with acute bronchiolitis. Among all RSV positive infants, $18.2 \%$ (16/88) had co-infection with pertussis. Demographic characteristics of infants with the positive pertussis-PCR and negative pertussis-PCR are shown in Table 2. Pertussispositive infants were significantly younger than pertussisnegative infants $(10 \pm 5.5 \mathrm{wk} v s .12 .5 \pm 6.6 \mathrm{wk} ; p=0.025)$. Pertussis-positive infants were not likely to have older siblings than pertussis-negative infants. Of the 44 pertussis-positive infants, $23(52.3 \%)$ were non-vaccinated, 15 (34.1\%) had received one dose of acellular pertussis vaccine, and $6(13.6 \%)$ had received two doses of the vaccine. None of pertussispositive infants had completed the primary pertussis 
Table 1 Respiratory pathogens identified in infants hospitalized with acute bronchiolitis $(n=172)$

\begin{tabular}{lcc}
\hline & $\mathrm{n}(\%)$ & $\begin{array}{l}\text { Co-infection } \\
\text { with B. pertussis, } \mathrm{n}(\%)\end{array}$ \\
\hline Respiratory syncytial virus & $88(51.1)$ & $16(18.2)$ \\
Rhinovirus & $55(31.9)$ & $11(20.0)$ \\
Bordetella pertussis & $44(25.6)$ & - \\
Parainfluenza virus & $15(8.7)$ & $1(6.6)$ \\
Influenza virus & $9(5.2)$ & $2(22.2)$ \\
Adenovirus & $8(4.6)$ & $4(50.0)$ \\
Human metapnomovirus & $7(4.0)$ & $2(28.5)$ \\
Human bocavirus & $7(4.0)$ & $3(42.8)$ \\
Human coronovirus & $5(3.5)$ & - \\
\hline
\end{tabular}

B. Pertussis Bordetella pertussis

vaccination series. Pertussis-PCR positivity was higher in infants who had received only one dose vaccine or who had been unvaccinated than those who had received two doses of the vaccine: $23(31.1 \%)$ of the 74 unvaccinated infants and $15(28.8 \%)$ of the 52 infants who had received one dose of acelluler pertussis vaccine were PCR-positive; while only 6 $(13 \%)$ of the 46 infants who received two doses were pertussis positive $(p=0.029)$.

There were no differences between pertussis-positive and pertussis-negative infants in terms of clinical and laboratory findings (Table 3). Paroxysmal coughing spells were present in $38.6 \%$ of pertussis-positive infants compared with $34.4 \%$ of pertussis-negative infants $(p=0.61)$. Of the pertussis-positive infants, $9.1 \%$ suffered from prolonged cough $v s .8 .6 \%$ of the pertussis-negative infants $(p=0.92)$. Frequency of whooping cough, post-tussive vomiting and apnea episodes were also not significantly different between the two groups. Pertussis was clinically suspected in only $7(15.9 \%)$ of the 44 infants who were found positive for pertussis by PCR. There was no statistically significant difference between pertussis-positive and pertussis negative infants according to the Wang clinical score at admission $(4.9 \pm 1.5$ vs. $5.2 \pm 2.5, p=0.689)$. Wheezing was present in $47.7 \%$ (21/44) of pertussis-positive infants compared with $48.4 \%(62 / 128)$ of pertussis-negative infants $(p=0.935)$. Oxygen saturation was also similar in the two groups $(95.6 \pm 3.8 v s .94 .0 \pm 4.8, p=0.066)$.

The overall disease severity score was also similar between the two groups $(6.5 \pm 1.4 v s .6 .9 \pm 1.6 ; p=0.095)$ (Table 3). The duration of hospital stay, the need or duration of supplemental oxygen and the need of intensive care treatment for the infants with pertussis were not significantly different to those with viral bronchiolitis. Only one pertussis-negative infant required transfer to the intensive care unit.

\section{Discussion}

Several studies have reported that pertussis was diagnosed by PCR in $7-23 \%$ of infants admitted to hospital with various respiratory tract infections including acute bronchiolitis and $36-67 \%$ of the pertussis cases were mixed infections with RSV or other respiratory viruses $[4,5,7-10,14]$. On the other hand, a study found only 1 of 166 children admitted to the hospital during RSV season was $B$. pertussis positive by PCR [6]. Another study found no $B$. pertussis positive cases by PCR in 204 infants aged $<18$ mo with bronchiolitis [15]. In the index study, however, $25.5 \%$ of young infants aged $<6$ mo hospitalized with acute bronchiolitis had a positive pertussis PCR and nearly two-thirds of the pertussis-positive infants were coinfected with respiratory viruses such as RSV. These findings indicate that pertussis respiratory virus co-infection is common in young infants hospitalized for acute bronchiolitis, which is in agreement with several studies [4, 5, 7-9].

Pertussis is a vaccine-preventable disease. However, even if the first dose of the pertussis vaccine provides partial protection, full infant protection may not be achieved until after
Table 2 Comparison of demographic characteristics and vaccination status of infants hospitalized with acute bronchiolitis: Pertussis-positive $v s$. pertussis-negative infants

\begin{tabular}{|c|c|c|c|}
\hline & $\begin{array}{l}\text { B. pertussis-positive } \\
(n=44)\end{array}$ & $\begin{array}{l}\text { B. pertussis-negative } \\
(n=128)\end{array}$ & $p$ \\
\hline Age, weeks (mean $\pm \mathrm{SD}$ ) & $10 \pm 5.5$ & $12.5 \pm 6.7$ & 0.025 \\
\hline \multicolumn{4}{|l|}{ Gender, n (\%) } \\
\hline $\begin{array}{l}\text { Male } \\
\text { Female }\end{array}$ & $\begin{array}{l}22(50) \\
22(50)\end{array}$ & $\begin{array}{l}81(63.2) \\
47(36.8)\end{array}$ & 0.12 \\
\hline Number of siblings, $\mathrm{n}$ (mean $\pm \mathrm{SD}$ ) & $2.07 \pm 0.9$ & $2.05 \pm 0.9$ & 0.93 \\
\hline $\begin{array}{l}\text { Number of people in the house, } \mathrm{n} \\
\quad(\text { mean } \pm \mathrm{SD}) \\
\text { Vaccination status, } \mathrm{n}(\%)\end{array}$ & $4.25 \pm 1.8$ & $4.54 \pm 1.5$ & 0.34 \\
\hline 0 dose & $23(31.1)$ & $51(68.9)$ & \multirow[t]{3}{*}{0.029} \\
\hline 1 dose & $15(28.8)$ & $37(71.2)$ & \\
\hline 2 doses & $6(13)$ & $40(87)$ & \\
\hline
\end{tabular}

B. pertussis Bordetella pertussis 
Table 3 Comparison of clinical and laboratory findings in pertussis-positive $v s$. pertussisnegative infants

\begin{tabular}{|c|c|c|c|}
\hline & $\begin{array}{l}\text { B. pertussis-positive } \\
(\mathrm{n}=44)\end{array}$ & $\begin{array}{l}\text { B. pertussis-negative } \\
(\mathrm{n}=128)\end{array}$ & $\begin{array}{l}p \\
\text { value }\end{array}$ \\
\hline \multicolumn{4}{|l|}{ Clinical characteristics } \\
\hline Paroxysmal cough, n (\%) & $17(38.6)$ & $44(34.4)$ & 0.610 \\
\hline Whooping, n (\%) & $13(29.5)$ & $35(27.3)$ & 0.779 \\
\hline Post tussive vomiting, n (\%) & $15(34.1)$ & $31(24.2)$ & 0.202 \\
\hline Apnea, n (\%) & $7(15.9)$ & $17(13.3)$ & 0.403 \\
\hline Prolonged cough (>14 d), n (\%) & $4(9.1)$ & $11(8.6)$ & 0.92 \\
\hline Wheezing, n (\%) & $21(47.7)$ & $62(48.4)$ & 0.935 \\
\hline Oxygen saturation $\%$, mean $\pm \mathrm{SD}$ & $95.6 \pm 3.8$ & $94.0 \pm 4.8$ & $0.066 \dagger$ \\
\hline Fever $>38^{\circ} \mathrm{C}, \mathrm{n}(\%)$ & $6(13.6)$ & $21(16.4)$ & 0.663 \\
\hline $\begin{array}{l}\text { Wang clinical score at admission, } \\
\text { mean } \pm \text { SD }\end{array}$ & $4.9 \pm 1.5$ & $5.2 \pm 2.5$ & $0.689 \dagger$ \\
\hline Duration of hospitalizition, mean $\pm \mathrm{SD}$ & $5.0 \pm 1.9$ & $5.8 \pm 2.5$ & $0.095 \dagger$ \\
\hline Supplemental oxygen therapy, n (\%) & $12(27.3)$ & $33(25.8)$ & 0.846 \\
\hline $\begin{array}{l}\text { Duration of oxygen therapy, days, } \\
\text { mean } \pm \text { SD }\end{array}$ & $0.6 \pm 1.0$ & $1.1 \pm 1.8$ & $0.202 \dagger$ \\
\hline $\begin{array}{l}\text { Number of infants who required intensive } \\
\text { care unit, } \mathrm{n}(\%)\end{array}$ & - & $1(0.78)$ & \\
\hline Overall disease severity score, mean $\pm \mathrm{SD}$ & $6.5 \pm 1.4$ & $6.9 \pm 1.6$ & $0.095 \dagger$ \\
\hline \multicolumn{4}{|l|}{ Laboratory findings } \\
\hline White blood cell $/ \mathrm{mm}^{3}$, mean $\pm \mathrm{SD}$ & $10,430 \pm 2700$ & $10,360 \pm 4200$ & $0.370 \dagger$ \\
\hline Lymphocytes $/ \mathrm{mm}^{3}$, mean $\pm \mathrm{SD}$ & $7180 \pm 1650$ & $5420 \pm 1650$ & $0.137 \dagger$ \\
\hline C-reactive protein, $\mathrm{mg} / \mathrm{dl}$, mean $\pm \mathrm{SD}$ & $0.6 \pm 0.8$ & $0.8 \pm 1.4$ & $0.306 \dagger$ \\
\hline
\end{tabular}

$†$ Mann-Whitney U test, B. pertussis Bordetella pertussis the completion of primary vaccination at 6 mo [16]. Acceptable immunity against pertussis is accomplished one month after the third dose of the vaccine [17]. Thus, unvaccinated or incompletely vaccinated young infants are highly susceptible to pertussis. In the index study, the majority of the pertussis-positive infants were unvaccinated or had received only one dose of acellular pertussis vaccine. None of the infants had completed the primary pertussis vaccination series. This may explain the high prevalence of $B$. pertussis in the present study group. Moreover, the prevalence of pertussis was higher in infants who had been unvaccinated $(31.1 \%)$ or who had received only one dose vaccine $(28.8 \%)$ than those who had received two doses of the vaccine $(13 \%)$.

Pertussis is underdiagnosed, especially in young infants hospitalized with lower respiratory tract infection [5, 7, 9]. In fact, in the present study, pertussis was clinically suspected in only $16 \%$ of the infants with acute bronchiolitis, who proved to be $B$. pertussis positive by PCR. The present study also shows that the viral bronchiolitis and pertussis cases could not be separated by clinical or non-specific laboratory findings. Some studies reported that infants who were pertussis-positive had paroxysmal cough and whooping cough episodes more often than infants who were pertussisnegative, which concluded that paroxysmal cough and whooping are important clinical signs for the diagnosis of pertussis [5, 10]. In the index study, however, most pertussis-positive infants presented no paroxysmal cough or whooping episodes. These findings suggest that the lack of paroxysmal cough or whooping cough is not sufficient enough to exclude the diagnosis of pertussis in young infants hospitalized with acute bronchiolitis, in agreement with several studies [7-9, 14]. Also, the typical features of viral bronchiolitis, or even the detection of respiratory viruses such as RSV, do not rule out pertussis [5, 9, 10]. For example, wheezing did not distinguish viral bronchiolitis and pertussis [7]. In the present study, wheezing was presented in only half of infants in whom RSV and/or other respiratory viral agents were detected, whereas similarly nearly half of the pertussispositive infants wheezed.

The clinical significance of $B$. pertussis infection in infants with acute bronchiolitis has not been clearly defined. Coinfection of $B$. pertussis with RSV was described to cause severe infections in 2 studies from the United States and Japan [18, 19]. However, newer studies by Greenberg et al. [5] and Crowcroft et al. [10] revealed that co-infection of pertussis and RSV did not adversely affect the outcome in patients admitted to the pediatric intensive care unit with various lower respiratory tract infections including acute bronchiolitis. Nuolivirta et al. [8] also suggests that there were no significant differences between $B$. pertussis positive and negative cases in the severity of the disease. A recent retrospective study from Israel by Abu Raya et al. [4] reported that young infants hospitalized with 
acute bronchiolitis in whom $B$. pertussis was detected had a milder disease severity at admission and during hospitalization than those in whom B. pertussis was not detected [4]. However, in the present prospective study which utilized the same disease severity scores with Abu Raya study, authors found that there were no significant differences in the severity scores at admission and during hospitalization for those with only viral infection compared with B. pertussis co-infection, and it is concluded that co-infection with pertussis did not affect the clinical outcome in infants hospitalized with acute bronchiolitis, which is in agreement with several other studies $[5,8,10]$. Nevertheless, early diagnosis and antibiotic treatment of pertussis is important because it is effective in eradicating B. pertussis from the nasopharynx, thus reducing the risk of transmission [20]. Otherwise, a missed diagnosis in pediatric wards or the pediatric intensive care units may lead to pertussis outbreaks among vulnerable young infants.

\section{Conclusions}

Pertussis-respiratory virus co-infection is common in young infants hospitalized for acute bronchiolitis. There are no consistent clinical criteria to distinguish pertussis concomitant with a viral bronchiolitis. The lack of paroxysmal cough or whooping does not rule out the diagnosis of pertussis. The detection of respiratory viruses, such as RSV, also does not rule out pertussis. To avoid underdiagnosis and to prevent possible pertussis outbreaks among vulnerable infants, pediatricians should be aware of these facts and should proceed with their diagnostic effort in order to detect pertussis in young infants hospitalized with acute bronchiolitis.

Acknowledgments The authors would like to thank the study staff at the Microbiology Laboratory for conducting the study, and the infants and their families for participating in the study.

Contributions All the authors were involved in screening and management of cases. SG analyzed and drafted the manuscript. ZK and SSA critically reviewed and finalized the manuscript. ZK will act as guarantor for this paper.

\section{Compliance with Ethical Standards}

Ethical Approval All procedures performed in studies involving human participants were in accordance with the ethical standards of the institutional and/or National Research Committee and with the 1964 Helsinki declaration and its later amendments or comparable ethical standards. The local ethics committee approved this study (reference number B.30.2.EGE.0.20.05.00/OY/1153/457).

\section{Conflict of Interest None.}

\section{Source of Funding None.}

\section{References}

1. Calvo C, Pozo F, García-García ML, et al. Detection of new respiratory viruses in hospitalized infants with bronchiolitis: a three-year prospective study. Acta Paediatr. 2010;99:883-90.

2. Mansbach JM, Piedra PA, Teach SJ, et al. Prospective multicenter study of viral etiology and hospital length of stay in children with severe bronchiolitis. Arch Pediatr Adolesc Med. 2012;166:700-6.

3. Berry M, Gamieldien J, Fielding BC. Identification of new respiratory viruses in the new millennium. Viruses. 2015;7: 996-1019.

4. Abu Raya B, Bamberger E, Kassis I, Kugelman A, Srugo I, Miron D. Bordetella pertussis infection attenuates clinical course of acute bronchiolitis. Pediatr Infect Dis J. 2013;32:619-21.

5. Greenberg D, Bamberger E, Ben-Shimol S, Gershtein R, Golan D, Srugo I. Pertussis is under diagnosed in infants hospitalized with lower respiratory tract infection in the pediatric intensive care unit. Med Sci Monit. 2007;13:CR475-80.

6. Siberry GK, Paquette NR, Ross TL, Perl TM, Valsamakis A. Low prevalence of pertussis among children admitted with respiratory symptoms during respiratory syncytial virus season. Infect Control Hosp Epidemiol. 2006;27:95-7.

7. Korppi M, Hiltunen J. Pertussis is common in nonvaccinated infants hospitalized for respiratory syncytial virus infection. Pediatr Infect Dis J. 2007;26:316-8.

8. Nuolivirta K, Koponen $\mathrm{P}, \mathrm{He} \mathrm{Q}$, et al. Bordetella pertussis infection is common in nonvaccinated infants admitted for bronchiolitis. Pediatr Infect Dis J. 2010;29:1013-5.

9. Cosnes-Lambe C, Raymond J, Chalumeau M, et al. Pertussis and respiratory syncytial virus infections. Eur J Pediatr. 2008;167: 1017-9.

10. Crowcroft NS, Booy R, Harrison T, et al. Severe and unrecognised: pertussis in UK infants. Arch Dis Child. 2003;88:802-8.

11. Ralston SL, Lieberthal AS, Meissner HC, et al. Clinical practice guideline: the diagnosis, management, and prevention of bronchiolitis. Pediatrics. 2014;134:e1474-502.

12. Wang EE, Milner RA, Navas L, Maj H. Observer agreement for respiratory signs and oximetry in infants hospitalized with lower respiratory infections. Am Rev Respir Dis. 1992;145:106-15.

13. Bamberger E, Srugo I, Abu Raya B, et al. What is the clinical relevance of respiratory syncytial virus bronchiolitis?: findings from a multi center prospective study. Eur J Clin Microbiol Infect Dis. 2012;31:3323-30.

14. Nelson WL, Hopkins RS, Roe MH, Glode MP. Simultaneous infection with Bordetella pertussis and respiratory syncytial virus in hospitalized children. Pediatr Infect Dis. 1986;5:540-4.

15. Walsh P, Overmeyer C, Kimmel L, et al. Prevalence of Bordetella pertussis and Bordetella parapertussis in Samples Submitted for RSV Screening. West J Emerg Med. 2008;9:135-8.

16. Juretzko P, von Kries R, Hermann M, Wirsing von König CH, Weil J, Giani G. Effectiveness of acellular pertussis vaccine assessed by hospital-based active surveillance in Germany. Clin Infect Dis. 2002;35:162-7.

17. Mooi FR, de Greeff SC. The case for maternal vaccination against pertussis. Lancet Infect Dis. 2007;7:614-24.

18. Moshal KL, Hodinka RL, McGowan KL. Concomitant viral and Bordetella pertussis infections in infants. Pediatr Infect Dis J. 1998;17:353-7.

19. Aoyama T, Ide Y, Watanabe J, Takeuchi Y, Imaizumi A. Respiratory failure caused by dual infection with Bordetella pertussis and respiratory syncytial virus. Acta Paediatr Jpn. 1996;38:282-7.

20. Tiwari T, Murphy TV, Moran J. Recommended antimicrobial agents for the treatment and postexposure prophylaxis of pertussis: 2005 CDC Guidelines. MMWR Recomm Rep. 2005;54:1-16. 